\title{
Tumor intracardíaco derecho: un caso sin precedentes
}

Úrsula Vargas-Gómez*

Juan Carlos González Altamirano**

* Servicio de cardiología. Hospital Nacional Carlos Alberto Seguín Escobedo. EsSalud Arequipa. Perú

** Servicio de cirugía cardíaca. Magister en bioética. Hospital III Yanahuara. EsSalud. Arequipa. Perú

\section{Correspondencia}

Úrsula Vargas-Gómez

ursula.mvg@gmail.com

\section{Recibido: 20/11/2019 \\ Aceptado: 16/01/2020 \\ En línea: 02/03/2020}

Citar como: Vargas-Gómez U, González-Altamirano JC. Tumor intracardíaco derecho: un caso sin precedentes. Rev Ecocar Pract (RETIC). 2020 (Mar); 3 (1): 41 -44. doi: 10.37615/retic.v3n1a11

Cite this as: Vargas-Gómez U, González Altamirano JC. Right intracardiac tumor: without precedents. Rev Ecocar Pract (RETIC). 2020 (Mar); 3 (1): $41-44$. doi: 10.37615/retic.v3n1a11.

\section{Palabras clave}

$\triangleright$ Tumor cardíaco

$\triangleright$ Enfermedad por lgG4

\section{Keywords}

$\triangleright$ Cardiac mass

$\triangleright \lg G 4$ disease

\section{Presentación del caso}

Mujer de 48 años, fumadora, que presenta 15 días antes de su hospitalización un primer episodio de angina típica de esfuerzo, recurrente y de curso progresivo, y que aparece finalmente en reposo y asociado a síntomas neurovegetativos y disnea.

En el electrocardiograma se vio infradesnivel del segmento ST con ondas T negativas en derivadas III y aVF (Figura 1), además de elevación de troponina T, por lo que se diagnostica como un síndrome coronario agudo. Al realizarle una ecocardiografía se encontró una imagen sugestiva de masa intracardíaca en aurícula derecha, por lo que se indagó la presencia de otras masas con estudios de imagen, marcadores tumorales y estudios funcionales, sin encontrar otro tumor que aparentara ser primario.

La ecocardiografía transesofágica dejó ver una masa de $28 \times 22$ mm en aurícula derecha adosada a anillo tricúspide y pared libre de $A D$, con flujo vascular interno, que no alteraba la función valvular (Figura 2, Vídeo 1 y Vídeo 2). La angiotomografía computarizada de arterias coronarias resaltó una masa que rodea la arteria coronaria derecha en su tercio medio y que, además, infiltra su luz (Figura 3 y Vídeo 3) La coronariografía (Figura 4) por cateterismo cardía- co confirmó una estenosis significativa de su luz, además de una coronaria izquierda sin estenosis en su trayecto. La paciente presentó un primer evento de taquicardia ventricular durante el estudio que requirió cardioversión eléctrica. El estudio funcional con FDG-glucosa (PET/TC) demostró una lesión hipodensa e hipermetabólica en el surco AV derecho, que engloba la porción distal de la arteria coronaria, altamente sospechosa de enfermedad neoformativa activa (Figura 5 y Figura 6).

Se discutió el caso en un Heart-Team y se llegó a la conclusión de estar frente a una neoformación de etiología maligna con compromiso vascular y, por tanto, altamente letal, por lo que se propuso el tratamiento quirúrgico, que fue aceptado por la paciente. En la sala de operaciones se realizó una biopsia por escisión con bypass safeno a la coronaria derecha, con los siguientes hallazgos: tumor sólido de $5 \times 4 \times 3 \mathrm{~cm}$ en AD que compromete a la arteria coronaria derecha y al anillo tricúspide, además de nódulo de aorta ascendente de $2 \mathrm{x}$ 2 duro.

La anatomía patológica mostraba una arteritis de coronaria derecha con marcada reducción de su lumen por hiperplasia fibrointimal y reemplazo parcial de la túnica media/túnica adventicia por un severo infiltrado linfoplasmocitario, con predominio de células plasmáticas, con presencia de acúmulos linfoides, y abundante fibrosis de patrón estoriforme, compatible con 
arteritis coronaria asociada a enfermedad relacionada a lgG4. La tumoración de pared de aorta fue descrita de forma similar, sugestiva de aortitis relacionada a lgG4 (Figura 7).

El diagnóstico se confirmó con el estudio inmunohistoquímico evidenciando lgG4 positivo en células plasmáticas (hasta 62 células lgG4/HPF) y relación lgG4/IgG0 40-50\%. Además, se obtuvo un valor de complemento C3 bajo, pero lgG4 en valor normal sérico, datos que en conjunto confirmaron el diagnóstico de una enfermedad por lgG4 en arteria coronaria derecha y aorta.

La evolución postoperatoria fue desfavorable por arritmia ventricular recurrente, encefalopatía hipóxico-isquémica post-RCP y sepsis, falleciendo a los 15 días de su intervención quirúrgica.

\section{Estudio por imagen}

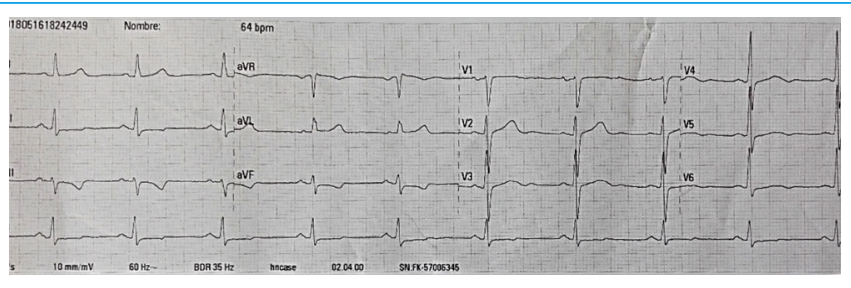

Figura 1. Electrocardiograma mostrando ritmo sinusal con alteración de la repolarización en cara inferior ( $\mathrm{D}$ |l y D III) sugestiva de isquemia

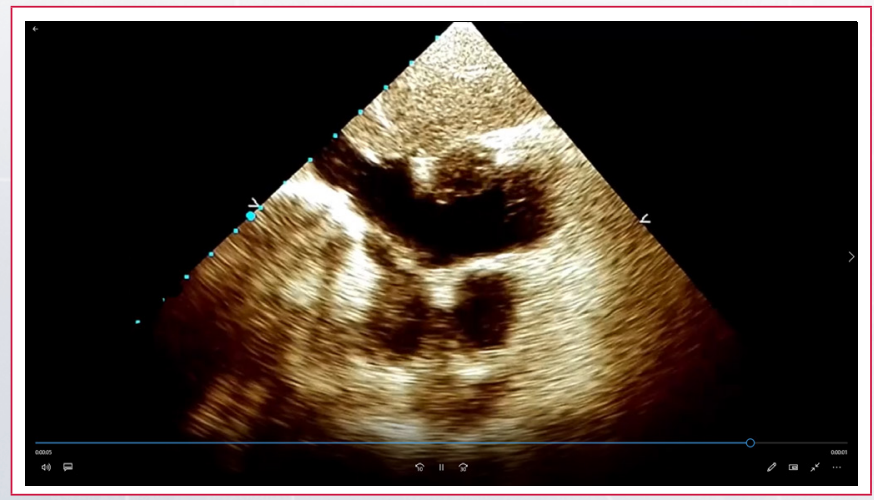

Vídeo 1. Ecocardiografía transesofágica bidimensional en ventana transgástrica a $0^{\circ}$, que muestra masa ovalada próxima a llegada de vena cava inferior y a válvula tricúspide

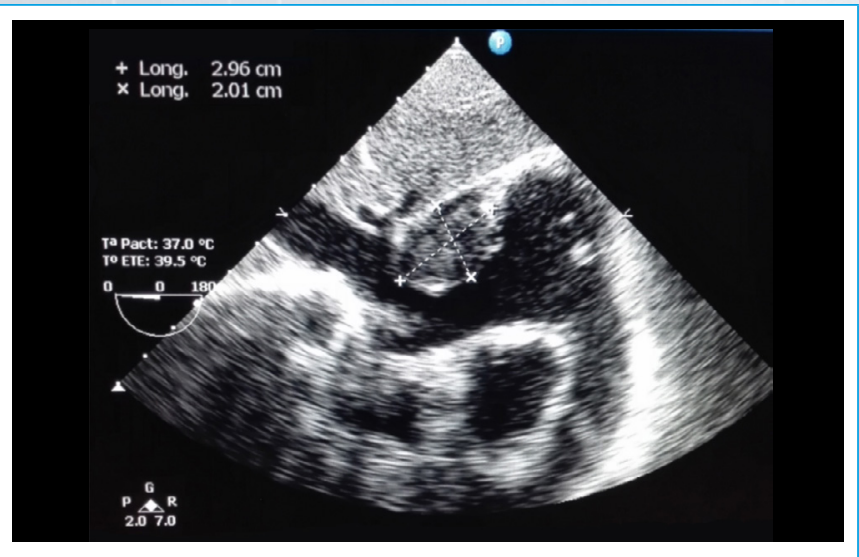

Figura 2. Imagen con las medidas de la masa

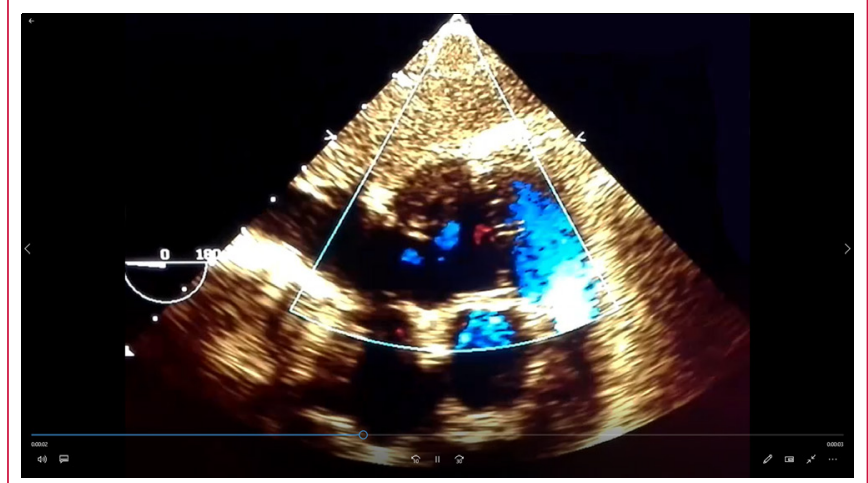

Vídeo 2. Ecocardiografía transesofágica bidimensional en ventana transgástrica a $0^{\circ}$, con Doppler color que muestra flujo en el interior de la masa

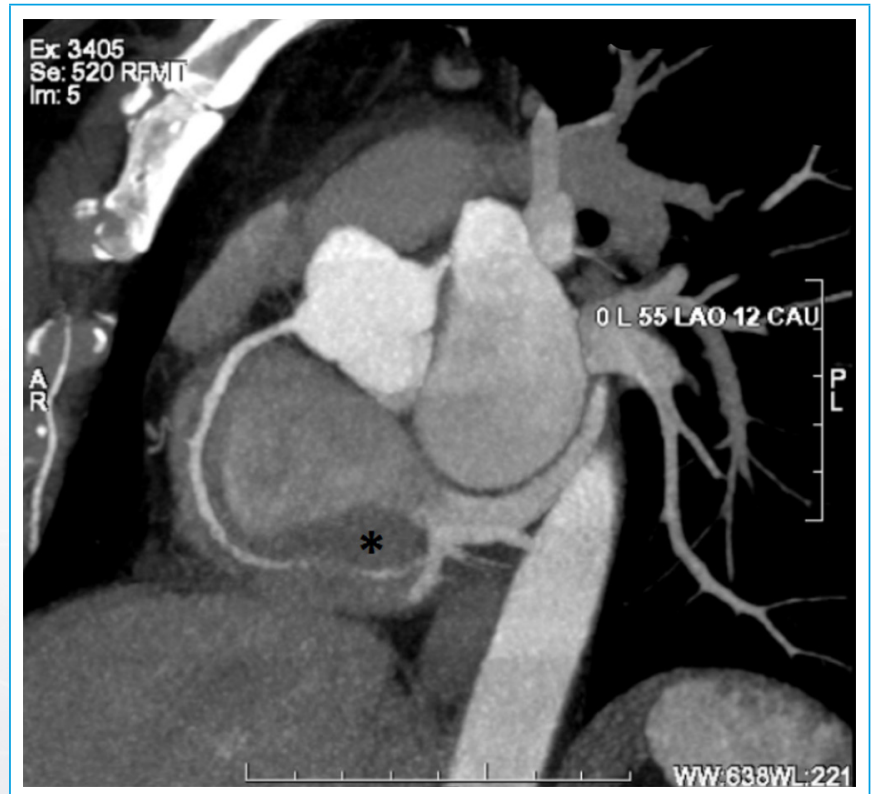

Figura 3. Angiotomografía con reconstrucción vascular y recorrido de arteria coronaria derecha donde se evidencia masa redondeada que rodea el tercio distal del vaso (asterisco)

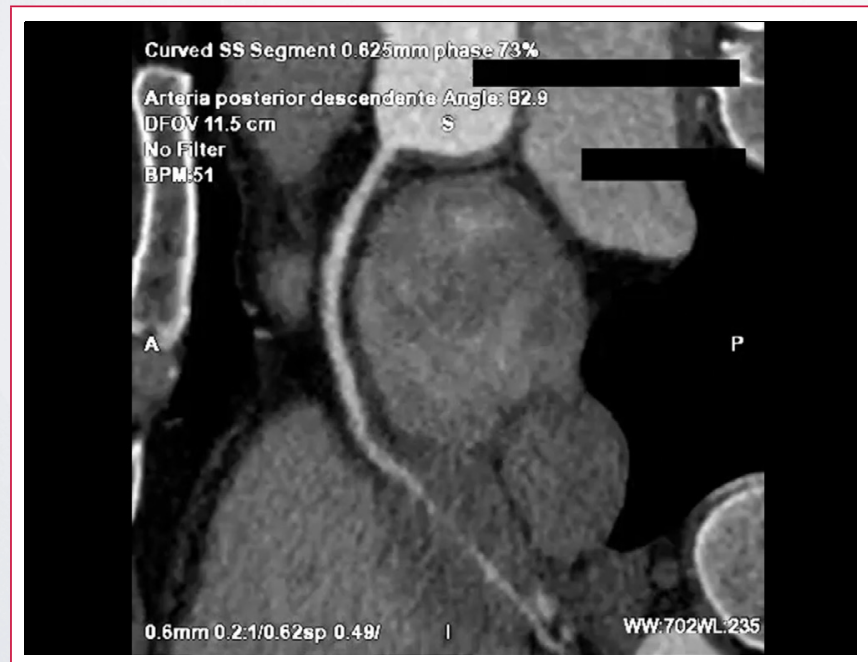

Vídeo 3. Angiotomografía con reconstrucción vascular y recorrido de arteria coronaria derecha donde se evidencia masa redondeada que rodea el tercio distal de la misma (asterisco) 


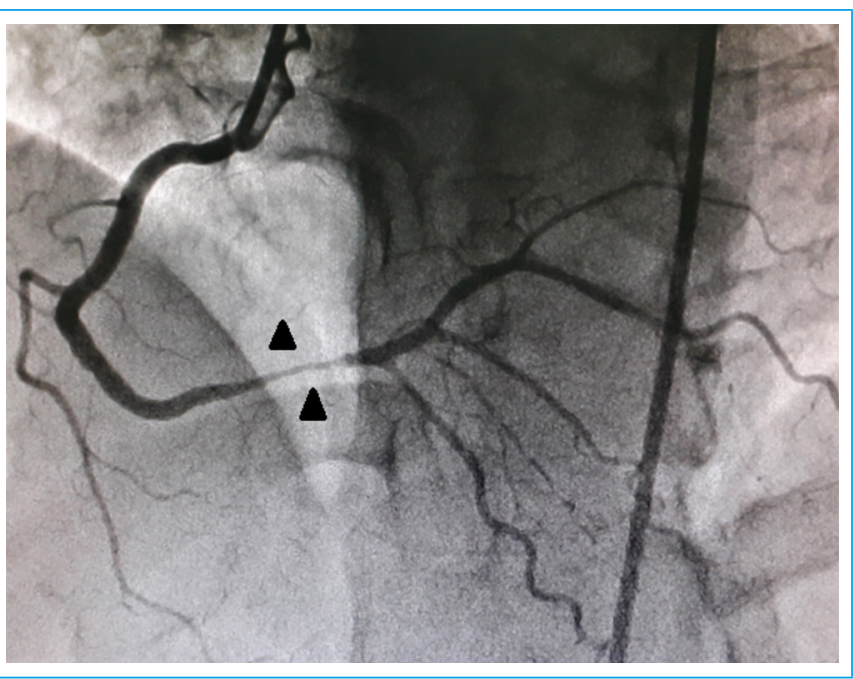

Figura 4. Coronariografía diagnóstica en vista oblicua anterior derecha que muestra la arteria coronaria derecha con buen calibre y desarrollo y sin una reducción significativa de la luz próxima a la rama descendente posterior (puntas de flecha)

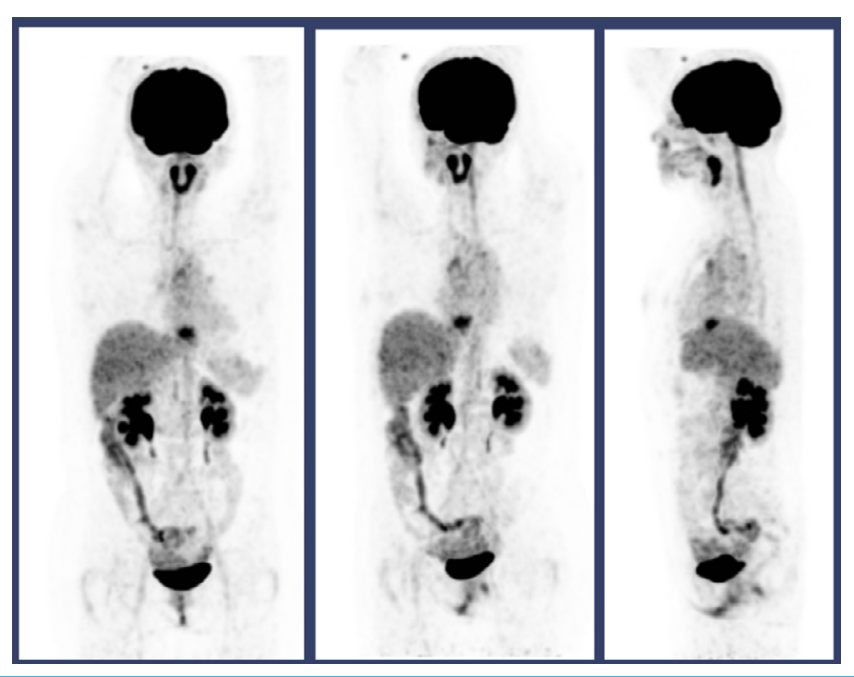

Figura 5. Tomografía por emisión de positrones con tomografía multicorte (PET/TC) con reconstrucción de captación normal de contraste (FDGglucosa) en cerebro, tejido tiroideo y sistema genitourinario. Existe una lesión hipermetabólica en surco auriculoventricular derecho en contacto con la aurícula derecha

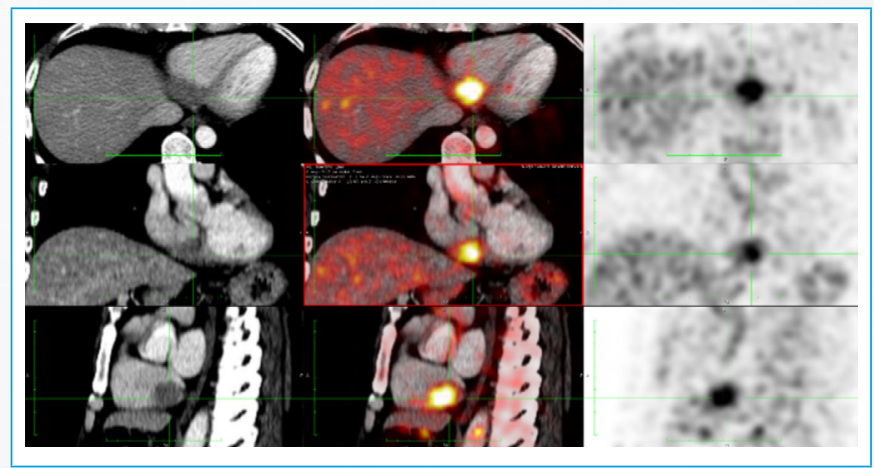

Figura 6. PET/TC que muestra una masa hipermetabólica en surco aurlculoventricular derecho alcanzado diámetro de $33 \times 23$ mm y SUV máx de 5,4 que incrementa en control tardío a 6,9. Este hallazgo es altamente sugestivo de enfermedad neoformativa activa (cortesía del Hospital Nacional Guillermo Almenara Irigoyen)

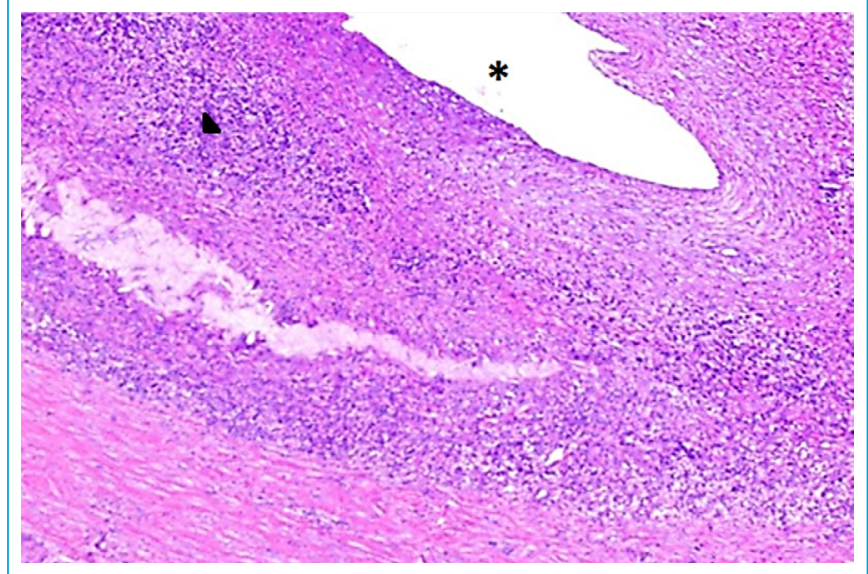

Figura 7. Anatomía patológica de tumoración en surco auriculoventricular que muestra luz de arteria coronaria derecha (asterisco), hiperplasia fibrointimal y reemplazo parcial de la túnica media/túnica adventicia por severo infiltrado linfoplasmocitario (punta de flecha) a predominio de células plasmáticas, con presencia de patrón estoriforme (cortesía de EsSalud-Servicio de Anatomía Patológica de Instituto Nacional del Corazón. Lima, Perú)

\section{Discusión}

Los tumores cardíacos primarios son raros, con una incidencia desde 0,001\% hasta $0,03 \%$. Las manifestaciones clínicas son diversas, dependiendo de su localización y estirpe. Entre ellos se encuentran neoplasias benignas y malignas que pueden desarrollarse a partir de cualquier tejido cardíaco ${ }^{(1-2)}$. Los tumores primarios más frecuentes son los mixomas ${ }^{(1,2)}$, que habitualmente son benignos y se localizan en la aurícula izquierda, mientras que los tumores malignos son más raros, ocupando cavidades derechas; siendo el sarcoma el más frecuente.

La enfermedad relacionada con IgG4 es una entidad recientemente nominada para definir diversas enfermedades caracterizadas por infiltración linfoplasmocítica, que frecuentemente afectan al páncreas (pancreatitis autoinmunitaria), y a las glándulas salivales (enfermedad de Mikulicz), aunque pueden comprometer casi cualquier estructura de la anatomía humana ${ }^{(3,4)}$. Se estima que la prevalencia de la enfermedad por lgG4 en Japón es de 2,63-10,2 casos por millón de habitantes ${ }^{(5)}$, con una incidencia de 336-1.300 casos nuevos por año(5).

\section{El diagnóstico se basa en:}

1. Lesión focal o difusa en uno o más órganos.

2. Concentraciones séricas de $\operatorname{lgG} 4>135 \mathrm{mg} / \mathrm{dl}$.

3. Histopatología compatible:

a) Infiltrado linfocitario y plasmocítico con fibrosis, sin infiltrado neutrófilo.

b) Infiltrado de plasmocitos IgG4 positivos mayor a 10 o proporción de células lgG $4 / \operatorname{lgG}>40 \%$.

c) Fibrosis estoriforme o remolino y flebitis obliterativa.

El diagnóstico se realiza con el cumplimiento de alguna de las siguientes combinaciones de criterios $^{(4)}$ :

- $1+2$.

- $1+3(a+b)$

- $2+3(a+b)$

- $3(a+b+c+d)$.

Los estudios de imagen revelan aumento de tamaño del órgano afectado o compromiso fibrosante regional. Los niveles séricos de lgG4 no se encuentran elevados en la totalidad de los pacientes en los que se llega a un diagnóstico por biopsia ${ }^{(4)}$. 
No existe un tratamiento específico, sin embargo, se utiliza un corticoide sistémico como primera línea, y se puede continuar con metotrexato, azatioprina o micofenolato, quedando rituximab para las recidivas ${ }^{(3-5)}$.

\section{Conclusión}

Se presenta el caso de una tumoración intracardíaca con características altamente sugerentes de malignidad primaria, manifestándose como un infarto de miocardio, que finalmente fue una entidad tumoral por lgG4 con compromiso de la arteria coronaria derecha, con comportamiento maligno y desenlace fatal. Se trata de un caso muy raro sin precedentes para el equipo médico quirúrgico tratante.

\section{Ideas para recordar}

- La ecocardiografía es la principal herramienta de ayuda al diagnóstico por imagen en la patología cardíaca.
- Los estudios funcionales son esenciales en la búsqueda y caracterización de una neoformación.

- La enfermedad por lgG4 es una entidad rara descrita recientemente y de la que se conoce aún poco, con compromiso de casi cualquier órgano y con comportamiento inesperado, aunque susceptible de tratamiento.

\section{Bibliografía}

1. Amano J, Nakayama J, Yoshimura Y, Ikeda U. Clinical classification of cardiovascular tumors and tumor-like lesions, and its incidences. Gen Thorac Cardiovas Surg 2013; 61 (8): 435-447.

2. Abad C. Tumores cardiacos (I). Generalidades. Tumores primitivos benignos. Rev Esp Cardiol 1998; 51 (1): 10-20.

3. Stone JH, et al. Editorial "IgG4-Related Disease." International journal of rheumatology 2013; ID 532612.

4. Umehara H, Okazaki K, Kawano M, et al. How to diagnose IgG4-related disease. Annals of the Rheumatic Diseases 2017; 76: e46.

5. Lendechy-Velázquez M, Hernández-Delgado A. Enfermedad relacionada con IgG4. Med Int Méx 2019; 35 (2): 313-320. 